\title{
High-energy effective theory for orbifold branes
}

\author{
Tetsuya Shiromizu ${ }^{(1,2,3)}$, Shunsuke Fujii ${ }^{(1)}$, Claudia de Rham ${ }^{(4)}$ and Hirotaka Yoshino ${ }^{(1)}$ \\ ${ }^{(1)}$ Department of Physics, Tokyo Institute of Technology, Tokyo 152-8551, Japan \\ ${ }^{(2)}$ Department of Physics, The University of Tokyo, Tokyo 113-0033, Japan \\ (3) Advanced Research Institute for Science and Engineering, Waseda University, Tokyo 169-8555, Japan and \\ (4) Ernest Rutherford Physics Building, McGill University, \\ 3600 rue University, Montreal, QC H3A 2T8, Canada
}

(Dated: September 24, 2018)

\begin{abstract}
We derive an effective theory on the orbifold branes of the Randall-Sundrum 1 (RS1) braneworld scenario in the presence of a bulk brane. We concentrate on the regime where the three branes are close and consider a scenario where the bulk brane collides with one of the orbifold branes. This theory allows us to understand the corrections to a low-energy approach due to the presence of higher velocity terms, coming from the Kaluza-Klein modes. We consider the evolution of gravitational waves on a cosmological background and find that, within the large velocity limit, the boundary branes recover a purely four-dimensional behavior.

PACS numbers: 98.80.Cq 04.50.+h 11.25.Wx
\end{abstract}

Recently, new scenarios have been suggested for a potential explanation for the creation of a hot big bang Universe [1]. The realization of such scenarios strongly depends on the possibility of a brane collision embedded in a higher-dimensional bulk. Motivated by this idea, an effective theory for a close brane system was addressed in Refs. 2, 3, 4, 5] (See Refs. 6, 7] for related issues). The procedure used in these papers has been developed in order to consider the high energy corrections to the low-energy theory within a close-brane regime. In this regime, we assume that the separations between the branes are much shorter than the bulk curvature scale. Recently cosmological perturbations was also discussed in [8], where an expansion in the brane velocities has instead been proposed. As a specific example, the effective theory on a bulk brane colliding with the orbifold branes has been studied in our previous paper [2]. The bulk brane has numerous specific features due to the reflection asymmetry across the bulk brane. In the ekpyrotic scenario [1], however, our Universe is assumed to be on one of the orbifold branes. Thus the aim of this report is to extend our previous study to the discussion for the boundary branes when such a bulk brane is.

For this purpose, we first describe the system in detail. Then we derive the effective theories on both orbifold branes and consider the evolution of tensor perturbations. We show that the tensor perturbations follow an equation which is different from what would be expected from a four-dimensional low-energy approach.

We consider the RS1 model [9] with an additional bulk brane between the two orbifold branes. The reflection symmetry is imposed for the two orbifold branes. On the other hand, no such reflection symmetry is imposed across the bulk brane [14] (See Refs. [10] for general issues on reflection asymmetric branes). The five dimensional metric of the bulk spacetime on both side of the bulk brane is

$$
d s_{ \pm}^{2}=e^{2 \varphi_{ \pm}(y, x)} d y^{2}+g_{\mu \nu}(y, x) d x^{\mu} d x^{\nu},
$$

where $y$ is the coordinate of extra dimension, and the index + (resp. - ) represents the bulk region located between the bulk brane and the positive (reps. negative) tension orbifold brane, which is located at $y=y_{+}$(resp. $\left.y=y_{-}\right)$. The location of the bulk brane is $y=y_{0}$ and we can suppose $y_{+}<y_{0}<y_{-}$without loss of generality. The gauge in (1) is chosen such that the branes are fixed to these given locations. The system is governed by the Einstein-Hilbert action with negative cosmological constant $\Lambda_{ \pm}=-6 / \kappa^{2} \ell_{ \pm}^{2}, \ell_{ \pm}$being the bulk Anti-de Sitter curvature. The brane action is the Nambu-Goto one with the four-dimensional action for matter fields localized on that brane. Due to the presence of boundary, the Gibbons-Hawking terms should also be introduced 11.

In the frame (1), the modified Einstein equation on the orbifold branes is written as [3, 12

$$
\begin{aligned}
{ }^{(4)} R_{\nu}^{\mu}\left(y_{I}\right)= & -\frac{4}{\ell_{I}^{2}} \delta_{\nu}^{\mu}+K\left(y_{I}\right) K_{\nu}^{\mu}\left(y_{I}\right)+e^{-\varphi_{I}} \partial_{y} K_{\nu}^{\mu}\left(y_{I}\right) \\
& +\left.e^{-\varphi_{I}} D^{\mu} D_{\nu} e^{\varphi_{I}}\right|_{y_{I}},
\end{aligned}
$$

where $K_{\nu}^{\mu}$ is the extrinsic curvature defined by $K_{\nu}^{\mu}=$ $\frac{1}{2} e^{-\varphi_{I}} g^{\mu \alpha} \partial_{y} g_{\alpha \nu}$ and the index $I= \pm$ represents the positive or negative tension brane. $D_{\mu}$ is the covariant derivative with respect to the induced metric $g_{\mu \nu}\left(y_{I}, x\right)$ on the brane. For the orbifold branes, the presence of the reflection symmetry allows us to specify uniquely the extrinsic curvature $K_{\nu}^{\mu}\left(y_{I}\right)$ through the Israël matching conditions [13]. These junction conditions relate the extrinsic curvature to the stress energy tensor ${ }^{(I)} T_{\nu}^{\mu}$ of matter fields localized on the brane:

$$
K_{\nu}^{\mu}\left(y_{I}\right)=-\frac{1}{\ell_{I}} \delta_{\nu}^{\mu}-I \frac{\kappa^{2}}{2}\left({ }^{(I)} T_{\nu}^{\mu}-\frac{1}{3} \delta_{\nu}^{\mu(I)} T\right) .
$$

In what follows, we will focus on the evaluation of $\partial_{y} K_{\nu}^{\mu}\left(y_{I}\right)$ in (2), which is the only unknown quantity. Since we are interested in a close-brane limit, we will use a Taylor expansion for the current system. We first note 
that, using Taylor expansion around $y=y_{I}$ and the recursive relation $\partial_{y}^{n} K_{\nu}^{\mu}\left(y_{I}\right)=\hat{O}_{I} \partial_{y}^{n-2} K_{\nu}^{\mu}\left(y_{I}\right)\left(y_{I}-y_{0}\right)^{-2}$, $K_{\nu}^{\mu}\left(y_{0}\right)$ can be written as [2, 4, 5 ]

$$
\begin{aligned}
{ }^{I} K_{\nu}^{\mu}\left(y_{0}\right)= & \cosh \sqrt{\hat{O}_{I}} K_{\nu}^{\mu}\left(y_{I}\right) \\
& +\left(y_{0}-y_{I}\right) \frac{\sinh \sqrt{\hat{O}_{I}}}{\sqrt{\hat{O}_{I}}} \partial_{y} K_{\nu}^{\mu}\left(y_{I}\right),
\end{aligned}
$$

where the operator $\hat{O}_{I}$ is defined below. This gives us the following expression for the derivative of the extrinsic curvature:

$$
\begin{aligned}
e^{-\varphi_{I}} \partial_{y} K_{\nu}^{\mu}\left(y_{I}\right)= & \frac{I}{d_{I}} \frac{\sqrt{\hat{O}_{I}}}{\sinh \sqrt{\hat{O}_{I}}}\left({ }^{I} K_{\nu}^{\mu}\left(y_{0}\right)\right. \\
& \left.-\cosh \sqrt{\hat{O}_{I}} K_{\nu}^{\mu}\left(y_{I}\right)\right)
\end{aligned}
$$

where $d_{I}$ represents the distance between the bulk brane and the orbifold brane:

$$
d_{I} \equiv e^{\varphi_{I}(x)}\left|y_{0}-y_{I}\right|
$$

and ${ }^{ \pm} K_{\nu}^{\mu}\left(y_{0}\right):=\lim _{\epsilon \rightarrow 0} K_{\nu}^{\mu}\left(y_{0} \mp \epsilon\right)$. The action of the operators $\hat{O}$ on any four-dimensional symmetric tensors $S_{\mu \nu}$ are defined by

$$
\begin{aligned}
\hat{O}_{I J} \equiv & \left|\left(y_{0}-y_{I}\right)\left(y_{0}-y_{J}\right)\right| \hat{U}_{I J} \quad I, J= \pm \\
\hat{U}_{I J} S_{\nu}^{\mu}= & D^{\mu} e^{\varphi_{I}} D_{\alpha} e^{\varphi_{J}} S_{\nu}^{\alpha}+D_{\nu} e^{\varphi_{I}} D_{\alpha} e^{\varphi_{J}} S^{\mu \alpha} \\
& -D_{\alpha} e^{\varphi_{I}} D^{\alpha} e^{\varphi_{J}} S_{\nu}^{\mu} .
\end{aligned}
$$

For the brevity, we used the notation of $\hat{O}_{I I}=\hat{O}_{I}$. In general, $\left|D_{\mu} d_{I}\right|$ is of order $\left|D_{\mu} d_{I}\right|=\mathcal{O}\left(d_{I}^{0}\right)$, (See Refs. 4, 5] for details). The operator $\hat{O}_{I J}$ is therefore of the same order: $\hat{O}_{I J}=\mathcal{O}\left(D d_{I} D d_{J}\right)=\mathcal{O}\left(d_{I}^{0} d_{J}^{0}\right)$. Note that the operator $\hat{O}_{I J}$ here is defined with respect to the orbifold brane metric.

In the expression (5), ${ }^{I} K_{\nu}^{\mu}\left(y_{0}\right)$ is still unknown quantity. Let then decompose ${ }^{I} K_{\nu}^{\mu}\left(y_{0}\right)$ into two parts as

$$
{ }^{I} K_{\nu}^{\mu}\left(y_{0}\right)=-I \frac{1}{2} \Delta K_{\nu}^{\mu}\left(y_{0}\right)+\bar{K}_{\nu}^{\mu}\left(y_{0}\right),
$$

where $\Delta K_{\nu}^{\mu}\left(y_{0}\right)$ is the part which can be determined by the junction condition at $y=y_{0}$ :

$$
\begin{aligned}
\Delta K_{\nu}^{\mu}\left(y_{0}\right) & :={ }^{-} K_{\nu}^{\mu}\left(y_{0}\right)-{ }^{+} K_{\nu}^{\mu}\left(y_{0}\right) \\
& =\left(\frac{1}{\ell_{+}}-\frac{1}{\ell_{-}}\right) \delta_{\nu}^{\mu}-\kappa^{2}\left({ }^{(0)} T_{\nu}^{\mu}-\frac{1}{3} \delta_{\nu}^{\mu(0)} \mathbb{T}(1) 0\right)
\end{aligned}
$$

In the above ${ }^{(0)} T_{\nu}^{\mu}$ is the energy-momentum tensor of matter fields localized on the bulk brane. $\bar{K}_{\nu}^{\mu}\left(y_{0}\right)$ is the averaged part (or "asymmetric" term) defined by

$$
\bar{K}_{\nu}^{\mu}\left(y_{0}\right):=\frac{1}{2}\left({ }^{+} K_{\nu}^{\mu}\left(y_{0}\right)+{ }^{-} K_{\nu}^{\mu}\left(y_{0}\right)\right) .
$$

Using the result of Ref. [2], in the close-brane regime, the asymmetric term can be expressed as:

$$
\bar{K}_{\nu}^{\mu}\left(y_{0}\right)=\hat{L} Z_{\nu}^{\mu},
$$

where

$$
\hat{L}=-\left[\frac{1}{d_{-}} \frac{\sqrt{\hat{O}_{-}}}{\tanh \sqrt{\hat{O}_{-}}}+\frac{1}{d_{+}} \frac{\sqrt{\hat{O}_{+}}}{\tanh \sqrt{\hat{O}_{+}}}\right]^{-1}
$$

and

$$
\begin{array}{r}
Z_{\nu}^{\mu} \equiv \sum_{I= \pm}\left[\left.\frac{I}{d_{I}} D^{\mu} D_{\nu}\right|_{y_{0}} d_{I}-\frac{1}{d_{I}} \frac{\sqrt{\hat{O}_{I}}}{\sinh \sqrt{\hat{O}_{I}}} K_{\nu}^{\mu}\left(y_{I}\right)\right. \\
\left.-\frac{I}{2 d_{I}} \frac{\sqrt{\hat{O}_{I}}}{\tanh \sqrt{\hat{O}_{I}}} \Delta K_{\nu}^{\mu}\left(y_{0}\right)\right] .
\end{array}
$$

In Eqs. (13) and (14), the operator $\hat{O}_{I}$ is defined on the bulk brane as $\hat{O}_{I}:=\left(y_{0}-y_{I}\right)^{2} \hat{U}_{I I}$, where $D_{\mu}$ is the covariant derivative with respect to the bulk brane metric. Since we are working in a close-brane regime, $g_{\mu \nu}\left(y_{0}\right)=g_{\mu \nu}\left(y_{ \pm}\right)+\mathcal{O}(d)$, and then

$$
\left.\hat{O}\right|_{y_{0}}=\left.\hat{O}\right|_{y_{I}}+\mathcal{O}(d) .
$$

In the current approximation, we can therefore simply replace $\hat{O}$ in the expression for $\bar{K}_{\nu}^{\mu}\left(y_{0}\right)$ by the operator on the orbifold branes.

The remaining task to obtain the effective theory on the orbifold branes is to write down $\left.D^{\mu} D_{\nu} d_{I}\right|_{y_{0}}$ in terms of quantities on the orbifold branes. This can be performed using the Taylor expansion:

$$
\left.D^{\mu} D_{\nu} e^{\varphi_{I}}\right|_{y=y_{0}}=\left.\sum_{n \geq 0} \frac{\left(y_{J}-y_{0}\right)^{n}}{n !} \partial_{y}^{n}\left(D^{\mu} D_{\nu} e^{\varphi_{I}}\right)\right|_{y=y_{J}},
$$

with

$$
\left.\partial_{y}^{2 n}\left(D^{\mu} D_{\nu} e^{\varphi_{I}}\right)\right|_{y=y_{J}}=-\hat{U}_{J I} \hat{U}_{J}^{n-1} \partial_{y} K_{\nu}^{\mu}\left(y_{J}\right)
$$

and

$$
\left.\partial_{y}^{2 n-1}\left(D^{\mu} D_{\nu} e^{\varphi_{I}}\right)\right|_{y=y_{J}}=-\hat{U}_{J I} \hat{U}_{J}^{n-1} K_{\nu}^{\mu}\left(y_{J}\right) .
$$

Eqs. (17) and (18) are derived from the expression for derivative of the Christoffel symbol(See Ref. [4] for the detail). As a result, one has

$$
\begin{aligned}
\left.\frac{1}{d_{I}} D^{\mu} D_{\nu} d_{I}\right|_{y=y_{0}}=\left.\frac{1}{d_{I}} D^{\mu} D_{\nu} d_{I}\right|_{y=y_{J}} \\
\quad-\frac{1}{d_{I}} \hat{O}_{J I} \frac{\cosh \sqrt{\hat{O}_{J}}-1}{\hat{O}_{J}}\left(d_{J} R_{\nu}^{\mu}\left(y_{J}\right)-\left.D^{\mu} D_{\nu} d_{J}\right|_{y_{J}}\right) \\
\quad-J \frac{1}{d_{I}} \hat{O}_{J I} \frac{\sinh \sqrt{\hat{O}_{J}}}{\sqrt{\hat{O}_{J}}} K_{\nu}^{\mu}\left(y_{J}\right),
\end{aligned}
$$


where we used the equation

$$
\partial_{y} K^{\mu}{ }_{\nu}=e^{\varphi(4)} R^{\mu}{ }_{\nu}-D^{\mu} D_{\nu} e^{\varphi}+\mathcal{O}(d) .
$$

Substituting Eq. (19) into Eq. (12) (with Eq. (9)), we can write down the extrinsic curvature on the bulk brane in terms of the quantities on the orbifold branes. Then we can compute the derivative of the extrinsic curvature on the orbifold branes, $\partial_{y} K_{\nu}^{\mu}\left(y_{I}\right)$, from Eq. (5). All ingredients for the derivation of the close-brane effective theory (2) are therefore presented.

But first, one may concentrate on the trace part of the four-dimensional Ricci tensor which is determined through the Hamiltonian constraint as

$$
{ }^{(4)} R\left(y_{I}\right)=-K_{\beta}^{\alpha}\left(y_{I}\right) K_{\alpha}^{\beta}\left(y_{I}\right)+K^{2}\left(y_{I}\right)-\frac{12}{\ell_{I}^{2}}=\mathcal{O}\left(d^{0}\right) .
$$

Then it is turned out to be negligible compared to the traceless part of the Ricci tensor because the traceless part is order of $\mathcal{O}\left(d^{-1}\right)$.

The equation for the radion may be derived using the tracelessness property of the projected Weyl tensor $E_{\mu \nu}\left(y_{I}\right):={ }^{(5)} C^{y}{ }_{\mu y \nu}\left(y_{I}\right)$. On the orbifold branes, $E_{\nu}^{\mu}\left(y_{I}\right)$ becomes [12]

$$
\begin{aligned}
E_{\nu}^{\mu}\left(y_{I}\right)= & -e^{-\varphi_{I}} \partial_{y} K_{\nu}^{\mu}\left(y_{I}\right)-\left.e^{-\varphi_{I}} D^{\mu} D_{\nu} e^{\varphi_{I}}\right|_{y_{I}} \\
& +\mathcal{O}\left(d^{0}\right) .
\end{aligned}
$$

Using the fact of $E_{\mu}^{\mu}\left(y_{I}\right)=0$, we obtain the Klein-Gordon equation for the radion $d_{I}$ as

$$
\begin{aligned}
\left.D^{2} d_{I}\right|_{y_{I}}= & -I \delta_{\mu}^{\nu} \frac{\sqrt{\hat{O}_{I}}}{\sinh \sqrt{\hat{O}_{I}}}\left({ }^{I} K_{\nu}^{\mu}\left(y_{0}\right)\right. \\
& \left.-\cosh \sqrt{\hat{O}_{I}} K_{\nu}^{\mu}\left(y_{I}\right)\right) .
\end{aligned}
$$

Finally the effective theory on the orbifold branes is derived as

$$
\begin{aligned}
& { }^{(4)} G_{\nu}^{\mu}\left(y_{I}\right)=\frac{1}{d_{I}}\left(\delta_{\beta}^{\mu} \delta_{\nu}^{\alpha}-\delta_{\nu}^{\mu} \delta_{\beta}^{\alpha}\right)\left[\left.D^{\beta} D_{\alpha} d_{I}\right|_{y_{I}}\right. \\
& \left.+I \frac{\sqrt{\hat{O}_{I}}}{\sinh \sqrt{\hat{O}_{I}}}\left({ }^{I} K_{\alpha}^{\beta}\left(y_{0}\right)-\cosh \sqrt{\hat{O}_{I}} K_{\alpha}^{\beta}\left(y_{I}\right)\right)\right],
\end{aligned}
$$

where ${ }^{I} K_{\nu}^{\mu}\left(y_{0}\right)$ can be expressed in terms of $Z_{\nu}^{\mu}$ and $\Delta K_{\nu}^{\mu}\left(y_{0}\right)$ using Eqs. (9) and (12). The tensor $Z_{\nu}^{\mu}$ can be written in the terms of $\Delta K_{\nu}^{\mu}\left(y_{0}\right)$ and $K_{\nu}^{\mu}\left(y_{I}\right)$. For that we substitute Eq. (19) for $\left.\frac{1}{d_{I}} D^{\mu} D_{\nu} d_{I}\right|_{y=y_{0}}$ into the expression (14) for $Z_{\nu}^{\mu}$ and then

$$
\begin{aligned}
Z_{\nu}^{\mu} & =\sum_{J= \pm} \frac{1}{d_{j}}\left[J \left\{\left.D^{\mu} D_{\nu} d_{J}\right|_{y_{I}}-I \hat{O}_{I J} \frac{\sinh \sqrt{\hat{O}_{I}}}{\sqrt{\hat{O}_{I}}} K_{\nu}^{\mu}\left(y_{I}\right)\right.\right. \\
& \left.-\hat{O}_{I J} \frac{\cosh \sqrt{\hat{O}_{I}}-1}{\hat{O}_{I}}\left(d_{I} R_{\nu}^{\mu}\left(y_{I}\right)-\left.D^{\mu} D_{\nu} d_{I}\right|_{y_{I}}\right)\right\} \\
& \left.-\frac{\sqrt{\hat{O}_{J}}}{\sinh \sqrt{\hat{O}_{J}}} K_{\nu}^{\mu}\left(y_{J}\right)-J \frac{\sqrt{\hat{O}_{J}}}{2 \tanh \sqrt{\hat{O}_{J}}} \Delta K_{\nu}^{\mu}\left(y_{0}\right)\right] .
\end{aligned}
$$

The effective theory (24) is the main result of this brief report and may be used for any studies within the closebrane regime. In particular this regime is of interest if one considers a bulk brane colliding with a boundary brane, and wishes to understand the propagation of the perturbations throughout this process. As we shall see below the behavior is different from the low-energy prescription.

As a concrete example, we consider the branes to be empty at the background level (except for a canonical tension) such that ${ }^{(\hat{I})} T_{\nu}^{\mu}=0$, for $\hat{I}= \pm, 0$. We then study the evolution of tensor perturbations generated by small stress-energy tensor ${ }^{(\hat{I})} \delta T_{j}^{i}$ on the branes at the perturbed level. The sources ${ }^{(\hat{I})} \delta T_{j}^{i}$ are taken to be tensor-like such that ${ }^{(\hat{I})} \delta T_{j}^{j}=0$ and ${ }^{(\hat{I})} \delta T_{j, i}^{i}=0$. We take the metric on the orbifold branes to be

$$
\begin{aligned}
d s^{2} & =-d t_{I}^{2}+a_{I}^{2}(t)\left(\delta_{i j}+h_{i j}\left(y_{I}\right)\right) d x^{i} d x^{j} \\
& =a_{I}^{2}(\tau)\left[-d \tau_{I}^{2}+\left(\delta_{i j}+h_{i j}\left(y_{I}\right)\right) d x^{i} d x^{j}\right],
\end{aligned}
$$

where $t_{I}$ and $\tau_{I}$ are the cosmic time and conformal time on the orbifold branes. We may point out that $\hat{O}_{+}$and $\hat{O}_{-}$commute for linear perturbations as pointed out in [2]. The linear part of Ricci tensor is

$$
\begin{aligned}
\delta R_{j}^{i}\left(y_{I}\right)= & \frac{1}{d_{I}} \delta\left(D^{i} D_{j} d_{I}\right)+I \frac{\sqrt{O}_{I}}{\sinh \sqrt{\hat{O}}_{I}}\left(\delta^{I} K_{j}^{i}\left(y_{0}\right)\right. \\
& \left.-\cosh \sqrt{\hat{O}_{I}} \delta K_{j}^{i}\left(y_{I}\right)\right)
\end{aligned}
$$

which leads us to the linearized equation for the gravitational waves $h_{j}^{i}\left(y_{ \pm}\right)$on the orbifold branes after long but straight-forward calculations:

$$
\hat{\ominus}_{ \pm} h_{j}^{i}\left(y_{ \pm}\right)=-\kappa^{2} a_{ \pm}^{2} \Omega_{ \pm}{ }^{(\mathrm{eff}, \pm)} \delta T_{j}^{i},
$$

where

$$
\begin{gathered}
\hat{\ominus}_{ \pm}:=\eta^{\mu \nu} \partial_{\mu} \partial_{\nu}-\frac{1}{U_{ \pm}} \frac{d_{+}^{\prime} d_{-}^{\prime}}{d_{+} d_{-}}\left(\frac{1}{\tanh \dot{d}_{+}}+\frac{1}{\tanh \dot{d}_{-}}\right) \partial_{\tau_{ \pm}} \\
U_{ \pm}=\frac{\dot{d}_{ \pm}}{d_{ \pm}}\left(\frac{1}{\tanh \dot{d}_{+}}+\frac{1}{\tanh \dot{d}_{-}}\right) \pm \frac{1}{\sinh \dot{d}_{ \pm}}\left(\frac{\dot{d}_{+}}{d_{+}}-\frac{\dot{d}_{-}}{d_{-}}\right) \\
\Omega_{ \pm}=\frac{1}{U_{ \pm}} \frac{\dot{d}_{+}}{d_{+}} \frac{\dot{d}_{-}}{d_{-}}
\end{gathered}
$$

and the effective stress-energy ${ }^{(\mathrm{eff}, \pm)} \delta T_{j}^{i}$ is given in terms of the stress-energy tensors ${ }^{(\hat{I})} \delta T_{j}^{i}$ on the branes:

$$
\begin{aligned}
{ }^{(\mathrm{eff}, \pm)} \delta T_{j}^{i}= & \left(1+\frac{1}{\tanh \dot{d}_{+} \tanh \dot{d}_{-}}\right)^{( \pm)} \delta T_{j}^{i} \\
& +\frac{1}{\sinh \dot{d}_{+} \sinh \dot{d}_{-}}{ }^{(\mp)} \delta T_{j}^{i} \\
& +2{\frac{\cosh \dot{d}_{\mp}}{\sinh \dot{d}_{+} \sinh \dot{d}_{-}}}^{(0)} \delta T_{j}^{i} .
\end{aligned}
$$

In particular we may be interested in the large velocity limit $\left(\left|\dot{d}_{I}\right| \gg 1\right)$. In this limit, the linearized equation 
becomes

$$
\left(\eta^{\mu \nu} \partial_{\mu} \partial_{\nu}-\frac{d_{\mp}^{\prime}}{d_{\mp}} \partial_{\tau_{ \pm}}\right) h_{j}^{i}\left(y_{ \pm}\right)=-a_{ \pm}^{2} \kappa^{2} \frac{\dot{d}_{\mp}}{d_{\mp}}{ }^{ \pm)} \delta T_{j}^{i} .
$$

The perturbations on a given orbifold brane only depend on its own matter content and do not depend on the ones on the bulk brane and the other orbifold brane. Here note that the matter content of the other branes contribute at small velocities. This is a remarkable result since this high-energy theory reproduces an exact four-dimensional behavior without any backreaction from the presence of the other branes, which would not be the case in a lowenergy regime. In Eq. (33), one might be surprised by the presence of the quantity $\dot{d}_{\mp} / d_{\mp}$ rather than $\dot{d}_{ \pm} / d_{ \pm}$. But within the close-brane behavior, we may recall that $d_{ \pm}=$ $\dot{d}_{ \pm} \tau+\mathcal{O}\left(\tau^{2}\right)$, and hence $\dot{d}_{+} / d_{+} \sim \dot{d}_{-} / d_{-} \sim 1 / \tau$, which is precisely the factor expected from a four-dimensional theory [5].

As a consistency check, we may also consider the special case where the reflection symmetry is imposed by hand across the bulk brane. The two orbifold branes should be identified and one can set $d_{+}=d_{-}=: d$, $a_{+}=a_{-}=a$ and ${ }^{(+)} \delta T_{j}^{i}={ }^{(-)} \delta T_{j}^{i}=: \delta T_{j}^{i}$ so that the linearized equation for tensor perturbations becomes

$$
\begin{aligned}
& \left(\eta^{\mu \nu} \partial_{\mu} \partial_{\nu}-\frac{d^{\prime}}{d} \partial_{\tau_{ \pm}}\right) h_{j}^{i}\left(y_{ \pm}\right) \\
& \quad=-\kappa^{2} a^{2} \frac{\dot{d}}{d}\left(\frac{1}{\tanh \dot{d}} \delta T_{j}^{i}+\frac{1}{\sinh \dot{d}}^{(0)} \delta T_{j}^{i}\right)
\end{aligned}
$$

which is consistent with the result obtained in Ref. 5 which explored a two-brane system with the reflection symmetry.

Finally we summarize our current study. In a previous work we derived the effective theory for a bulk brane in the context of the RS1 braneworld model. In this brief report, we derived the effective theory for orbifold branes within the same setup. In the close-brane regime, the metric on the orbifold branes and the bulk brane are trivially related to each other. However, we would like to stress that the individual derivation of the effective theory for each brane is important for the physical interpretation on the time evolutions as well as for fixing the initial conditions. We have therefore derived separately the effective close-brane theory on the orbifold branes and have recovered the similar main features found for the bulk brane. The difference appears in the gravitational coupling. Nevertheless, within the largevelocity limit, the behavior of the branes becomes purely four-dimensional and decouples entirely from the other branes, which is not the case within a low-energy regime.

\section{Acknowledgements}

The work of TS was supported by Grant-in-Aid for Scientific Research from Ministry of Education, Science, Sports and Culture of Japan(No.13135208, No.14102004, No. 17740136 and No. 17340075) and the Japan-U.K. Research Cooperative Program. CdR is funded by a grant from the Swiss National Science Foundation.
[1] J. Khoury, B. A. Ovrut, P. J. Steinhardt and N. Turok, Phys. Rev. D64, 123522 (2001).

[2] C. de Rham, S. Fujii, T. Shiromizu and H. Yoshino, Phys. Rev. D72, 123522 (2005).

[3] T. Shiromizu, K. Koyama and K. Takahashi, Phys. Rev. D67, 104011 (2003).

[4] C. de Rham and S. Webster, Phys. Rev. D71, 124025 (2005).

[5] C. de Rham and S. Webster, Phys. Rev. D72, 64013 (2005).

[6] A. Neronov, JHEP 0111, 007(2001); D. Langlois, K. Maeda and D. Wands, Phys. Rev. Lett. 88, 181301 (2002); S. Kanno, M. Sasaki and J. Soda, Prog. Theor. Phys. 109, 357 (2003).

[7] U. Gen, A. Ishibashi and T. Tanaka Phys. Rev. D66, 023519 (2002); J. J. Blanco-Pillado, M. Bucher, S. Ghassemi and F. Glanois, Phys. Rev. D69, 103515 (2004).

[8] P. L. MacFadden and N. Turok, hep-th/0512123

[9] L. Randall and R. Sundrum, Phys. Rev. Lett. 83, 3370
(1999).

[10] A. C. Davis, S. C. Davis, W. B. Perkins and I. R. Vernon, Phys. Lett. B504, 254 (2001); B. Carter and J. P. Uzan, Nucl. Phys. B606, 45 (2001); K. Takahashi and T. Shiromizu, Phys. Rev. D70, 103507 (2004); L. A. Gergely, E. Leeper and R. Maartens, Phys. Rev. D70, 104025 (2004); I. R. Vernon and D. Jennings, JCAP 0507, 011 (2005).

[11] G. W. Gibbons and S. W. Hawking, Phys. Rev. D15, 2752 (1977).

[12] T. Shiromizu, K. Maeda and M. Sasaki, Phys. Rev. D62, 024012 (2000), T. Shiromizu and K. Koyama, Phys. Rev. D 67, 084022(2003) arXiv:hep-th/0210066.

[13] W. Israel, Nuovo Cim. B 44S10, 1 (1966) [Erratum-ibid. B 48 (1967 NUCIA,B44,1.1966) 463].

[14] The precise description of our setup is seen from the junction condition for the extrinsic curvature described below. 